\title{
Linear permutation polynomials with coefficients in a subfield
}

by

J. V. Brawlery (Clemson, S. O.), T. Carlirz* (Durham N. C), and Therase Vaugtran (Durham, N. C.)

To Professor Carl Ludwig Siegel

1. Introduction. Let $\mathrm{GT}\left(q^{n}\right)$ denote the finite field of order $q^{n}$, where $q=p^{r}$ for some $r>0$ and some prime $p$, and let $\mathrm{GF}\left(q^{m}\right)$ be a subfield of $\mathrm{GT}\left(q^{n}\right)$ so that $n=m s$ for somo integer $s, 1 \leqslant s \leqslant n$. If $f$ is any function from $\operatorname{GF}\left(q^{n}\right)$ to $G \mathrm{GE}\left(q^{n}\right)$ it is well-known that $f$ has a unique polynomial representation.

$$
f(x)=\sum_{i=0}^{\alpha^{n}=1} a_{i} x^{i}
$$

where the coefficients $a_{i} \in \mathrm{GN}\left(q^{n}\right)$. In case $f$ is a permatation of $\mathrm{GF}\left(q^{n}\right)$ the corresponding polynomial $f(x)$ is called a permutation polynomial. The set of all such permutation polynomials under composition modulo $x^{a^{n}}-x$ forms a group which is isomorphie to the symmetric group $S_{q^{n}}$. Those permutation polynomials of the form (1.1) whose coefficients $a_{i}$ are in $\left(\mathrm{KF}^{\prime}\left(q^{m}\right)\right.$ constitute a subgroup, the structure of which has been. determined by Carlitz and Hayes [2] as a semi-direct product of certain symmetric groups and cyclie groups. In this paper we consider an analogons situation for polynomials of the form.

$$
f(x)=\sum_{i=0}^{n} a_{i} x^{q^{l}}
$$

with coefficients $a_{i}$ in $\mathrm{GW}\left(q^{m}\right)$. Such polynomials (which represent a subal-

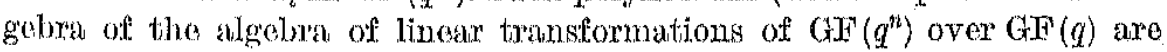
generalizations of the Oro polynomials [6], [7], where the coefficients $a_{i}$ are assumed to lie in $G(F)$. The set of all Ore polynomials under the modulo $x^{l^{n}}-x$ operations of addition and composition of functions, and sealar multiplication by elements of $G \mathrm{FF}(q)$, forms a commutative algebra over $\mathrm{GF}^{\prime}(q)$ which is isomorphic to $\mathrm{GF}(q)[x] /\left(x^{n}-1\right.$ ) (see [7]).

\footnotetext{
* Supported in part by NSF grant GP-17031.
} 
In order to gencralize Oro's work, put

$$
R_{m}=\left\{f(x)=\sum_{i=0}^{n-1} a_{i} x^{d^{i}} ; a_{i} \epsilon \operatorname{GF}\left(p^{m}\right)\right\} .
$$

Then $R_{n}$ under the above mentioned operations is an algebra over GF $(q)$ The case $m=1$ is that treated by Ore. In $\$ 2$ of the present paper we show that $R_{n}$ is isomorphic to the ring of $n \times n$ matrices over GE $(q)$ from which it follows that the group of units of $R_{n}$, the so-called Botti-Mathien group, is isomorphic to the general linear group Gr. $(n, q)$. (See [1], [3].) In $\S 3$ we prove that $R_{m}$ is isomorphic to the ring of $m \times m$ matrices with entries from the residue class $\operatorname{ring} \mathrm{GT}(q)[x] /\left(x^{s}-1\right)$. This includes Ore's result as well as that given in $\$ 2$ as special cases. Using this isomorphism it is easy to describe the group of units of $R_{m}$ as a direct product of subgroups in contrast to the Carlitz-Hayes result. This description and several interesting combinatorial results are contained in $\$ 4$.

2. Preliminaries. The ring of polynomials with coefficients in GF $(q)$ will be denoted by $\mathrm{GF}(q)[x]$. If $f(x) \in \mathrm{GF}(q)[x]$, the principal ideal generated by $f(x)$ is denoted by $(f(x))$, and the residue class ring consisting of the elements of $G F(q)[x]$ reduced modulo $f(x)$ is writton $G F(q)[x] /(f(x))$. Also if $S$ is any ring with identity and $k$ is any positive integer, the ring of $k \times \%$ matrices with elements from $S$ will be written as $(S)_{k}$, and $\mathrm{GL}(k, S)$ will denote the group of nonsingular $k \times 7$ matrices over $S$. In case $\$$ $=G \mathbb{F}\left(q^{n}\right)$ the notation GLL $\left(k, q^{n}\right)$ is used for $G \mathrm{~L}\left(n, S^{\prime}\right)$.

Consider the finite field $\mathrm{GF}^{\prime}\left(q^{n}\right)$ as a vector space of dimension $n$ over GF $(q)$. Let $L$ be the algebra of limear transformations of GF $\left(q^{2 n}\right)$ over $\operatorname{GF}(q)$. The set $R_{n}$ of all polynomials of the form

$$
f(x)=\sum_{i=0}^{n-1} a_{i} x^{q^{i}}
$$

with the coefficients $a_{i} \in$ GF $\left(q^{n}\right)$, equipped with the modulo $x x^{q^{n}}-x$ operations of addition and composition of functions and scalar multiplication. by elements of $\mathbf{G F}(q)$, is an algebra over GT $(q)$, which is in facti inomorphic to the algebra, $I$.

Tneoxwm 2.1. The algebras $R_{q_{2}}$ and $L$ are isomorphis.

Proof. For each $f(x) \in R_{m}$ let $f$ be the tunction from $\operatorname{ar}\left(q^{n}\right)$ to $\operatorname{ar}\left(q^{n}\right)$ defined by substitution and lot $\psi$ denote the mapping that takes $f(x)$ to $f_{i}$ Then for each $f(x) \in R_{n} \quad \psi(f(x)) \in L$ as $\quad(\xi+\eta)^{q^{i}}=\xi^{q^{i}}+\eta^{i^{i}}$ and $(\lambda \xi)^{q^{i}}=\lambda^{q^{i}} \xi^{q^{i}}=\lambda \xi^{q^{i}}$ for all integers $i>0$ and $\xi, \eta \in G \mathrm{~F}^{i}\left(q^{n}\right), \lambda \in \mathrm{GF}(q)$, i.e., $\psi: R_{n} \rightarrow L$. Moreover, it is immediate that

$$
\begin{gathered}
\psi(f(x)+g(x))=f+g \\
\psi(\lambda f(x))=\lambda f
\end{gathered}
$$

and

$$
\psi(f(g(x)))=f \circ g
$$

so that $\psi$ is an algebra homomorphism. $\psi$ is one-one by the remark in the first paragraph of $\xi \cdot 1$, and sinoe $\left|R_{n}\right|=\left(q^{n}\right)^{n}=q^{n^{2}}=|L|$, the proof is complete.

It follows immediately that

Conotiraxi 2.2. The algebra $R_{n}$ is isomorphic to $(\mathrm{GF}(q))_{n}$.

The Betti-Mathien group is by definition the group of units of $R_{n}$; henee we have reproved (see [1], [3])

Cororrary 2.3. The Botti-Mathien group is isomorphio to $\mathrm{GL}(n, q)$.

3. The algebra $R_{m}$. If $n=m s$, where $m$ and $s$ are positive integers, we define the algebra $R_{m}$ to be the set of all polynomials of the form

$$
f(x)=\sum_{i=0}^{n-1} a_{i} x^{q^{i}}
$$

with coefficients $a_{i} \in \mathrm{GE}\left(q^{m}\right) \subseteq \mathrm{GE}\left(q^{n}\right)$, equipped with addition and composition of functions and scalar multiplication by elements of GF $(q)$. When $m=n$, the algebra $R_{n}$ is, as we have seen, isomorphic to tho algebra of all linear transformations of $\mathrm{GT}\left(q^{n}\right)$ over $\mathrm{GF}(q)$. When $m=1$, the algebra $R_{1}$ is the algebra of polynomials studied by ore in [6], [7] who has shown that $R_{I}$ is isomorphic to the residue class $\operatorname{ring} \mathrm{GF}(q)[x] /\left(x^{n}-1\right)$. The next theorem is a gencralization of these results.

TIIEORIM 3.1. If $n=m s$, where $m$ and $s$ are positive integers, then the algebra $R_{m}$ is isomorphic to the algebra $\left(\mathrm{GF}(q)[x] /\left(x^{s}-1\right)\right)_{m}$, of $m \times m$ matrices with elements from the residue class ring $\mathrm{GF}(q)[x] /\left(x^{s}-1\right)$.

Proof. For convenience, let $S_{m}=\left(\mathrm{GE}(q)\left[x^{2}\right] /\left(x^{s}-1\right)\right)_{m}$. Fix any ordered basis $B=\left\{\beta_{1}, \beta_{2}, \ldots, \beta_{m}\right\}$ for $\operatorname{GF}\left(q^{m}\right)$ over $\mathrm{GF}(q)$. If $f(x)=\sum_{i=0}^{m-1} a_{i} x^{q^{i}}$ with coefficients $a_{i}$ in $G F\left(q^{m}\right)$, let $[f]_{B}$ denote the matrix in $(G \mathbb{F}(q))_{m}$ which represents tho lineas transormation $f(x)$ in the ordered basis $B$.

Wo first note that any eloment of $R_{m}$, say

may be rewrititen as

$$
g(x)=\sum_{i=0}^{n+1} a_{i} x^{i^{i}} ; \quad a_{i} \in \operatorname{GTE}\left(q^{m}\right)
$$

$$
g(x)=\sum_{i=0}^{s} \sum_{k=0}^{m-1} a_{i m+k} x^{2^{i n-1+k}} .
$$

If we let $g_{i}(x)=\sum_{l i m i t}^{m-2} a_{i m+h} x^{t^{k}}$ for $i=0,1, \ldots, s-1$, then we may write

$$
g(x)=\sum_{i=10}^{m-1} g_{i}\left(x^{q^{m i}}\right)
$$


On the other hand, any element $\not F$ of $S_{m}$ has the form $F=\left(f_{i j}(x)\right)$ (for $i, j=0,1, \ldots, m-1$ ), where each $f_{i j}(x)$ is a polynomial over GE $(q)$ of degree less than $s$, and we may rewrite the matrix $F$ as follows:

$$
F=F_{0}+W_{1} x+F_{2}^{\prime} x^{2}+\ldots+F_{s+1}^{\prime} x^{s-1}
$$

where each $F_{k}(k=0,1, \ldots, s-1)$ is an $m \times m$ matrix over GF $(q)$, and the $(i, j)$ the entry of $F_{k}$ is the coeffieient of $x^{k}$ in the polymomial $f_{i j}(x)$.

Now for each $F_{k}$, there exists a polynomial

$$
g_{k}(x)=\sum_{i=0}^{m-1} b_{i k^{2} x^{q^{i}}}
$$

such that $F_{k}$ is the matrix representing $g_{k}(x)$ in the ordered basis $B$, that is, $F_{k}=\left[g_{k}\right]_{B}$.

It is now fairly obvious how to define an isomorphism between $S_{m}$ and $R_{m}$. If $\not F$ is given by $(3.5)$ and the corresponding $g_{k}(x)$ are given by $(3.6)$, define a mapping $\varphi: S_{m} \rightarrow R_{m}$ by

$$
\varphi\left(F_{k} x^{k}\right)=g_{l i}\left(x^{k^{k m}}\right)
$$

where it is understood that map $\phi$ is to be extended linendy to all of $S_{m}$. It is elear that this is indeed a maj from $S_{m}$ to $R_{m}$, since every element of $R_{m}$ may be written in the form (3.4). Tridently addition and sealar multiplication by elements of $\mathrm{GF}(q)$ are preserved, and it follows from Theorem 2.1. that $\varphi$ is bijective. It remains only to show that the map $\varphi$ preserves composition. Suppose that $G=[g]_{B}$ and $H=[h]_{b}$ are any two matrices in $(G T(q))_{m}$. Then if $i$ and $k$ are positive integers less than $s$, and $i+k=j(\bmod s)$, we have

$$
\text { (3.8) } \quad p\left(G x^{i}\right) \circ p\left(H x^{k}\right)=g\left(x^{q^{i m}}\right) \circ h\left(x^{q^{k m}}\right)=g\left(h\left(x^{2^{k m}}\right)^{q^{i m}}\right)=g\left(h\left(x^{q^{j m}}\right)\right)
$$

since the coefficients of $h(x)$ are elements of $\mathrm{GF}\left(q^{m}\right)$. Since $G x^{i} H x^{h}=G H x^{j}$, and $p\left(G H x^{j}\right)=g\left(h\left(x^{q^{j m}}\right)\right)$, it follows that

$$
\varphi\left(G x_{i}^{i}\right) \circ p\left(H x^{i}\right)=p\left(G H\left(x^{j}\right)\right.
$$

and so $p$ preserves composition. Thus $p$ is an isomorphism. This completes the proof.

4. The group of units of $R_{n}$ and related results. Tin order tio characterize the group of units of $R_{m}$ we will use the following known fnets.

Lrimara 4.1. If $S$ is a commutative wing with $x$ whish has tho direct sum decomposition $S=\oplus \sum_{i=1}^{t} S_{i}$, then $(S)_{m}=\oplus \sum_{i=1}^{t}\left(S_{i}\right)_{m}$ and moreover $\mathrm{GL}(m, S)=\oplus \sum_{i=1}^{t} \mathrm{GL}\left(m, \mathbb{S}_{i}\right)$ so that

$$
\left|\mathrm{GL}\left(m, S^{\prime}\right)\right|=\prod_{i=1}^{t}\left|\mathrm{GL}\left(m, s_{i}\right)\right| .
$$

LEMINA 4.2. If $S=\mathrm{GF}(q)[x] /\left(P(x)^{e}\right)$ where $P(x)$ is an irreducible in $\mathrm{GF}(q)[x]$ of degree $d$, then

$$
|\mathrm{GL}(m, s)|=q^{c d m^{2}} \prod_{i=1}^{m}\left(1-q^{-i d}\right) .
$$

The proof of Lemma 4.1. is easy. As for Lemma 4.2, one can use the formula of MeDonald [5] onee it is noted that $S$ is a finite local ring. Basically, the proof uses the correspondence theorem for pings together with the facts that (i) $M=P(x) \cdot S$ is the unique maximal ideal of $S$, (ii) $S / M=\mathrm{GF}\left(q^{l}\right)$ and (iii) $A \epsilon(S)_{m}$ is nonsingular iff $\mu(A)=\left(\mu\left(a_{i j}\right)\right) \epsilon(S / M)_{m}$ is nonsingular where $\mu: S \rightarrow S / M$ is the natural homomorphism.

THEOREM 4.3. Let $S=\mathrm{GW}(q)[n] /\left(x^{s}-1\right)$, and suppose that

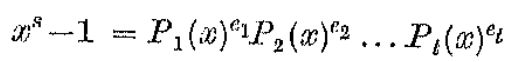

where the $P_{j}(x)$ are distinct irreducible elements of $\mathrm{GF}(q)[x]$, and the degree of $P_{j}(x)$ is $a_{j}$ for $j=1,2, \ldots$, t. Set $S_{j}=\mathrm{GW}(q)[x] /\left(P_{j}(x)^{c_{j}}\right)$. Then $\mathrm{GL}(m, \$)$ is isomorphic to the direct product of the set $\left\{\operatorname{GL}\left(m, S_{j}\right): j=1,2, \ldots, t\right\}$ and moreover

$$
\left|\mathrm{GI}_{1}(m, S)\right|=q^{m m^{2} s} \prod_{j=1}^{t} \prod_{i=1}^{m}\left(1-q^{-i d_{j}}\right)
$$

Proof. It is only necessary to note that $S=S_{1} \oplus S_{2} \oplus \ldots \oplus S_{t}$. Then by Lemma 4.1, GL $(m, S)$ is isomorphic to the direct product of the set $\left\{\operatorname{GL}\left(m, S_{j}\right): j=1,2, \ldots, t\right\}$. To get the equality (4.4), use Lemma 4.2 :

$$
\left|\mathrm{GL}\left(m, \xi_{j}\right)\right|=q^{e_{j} d_{j} m^{2}} \prod_{i=1}^{m}\left(1-q^{-i d_{j}}\right)
$$

and from Lemma 4.工,

$$
\begin{aligned}
\left|\mathrm{G} \mathrm{L}\left(m, s^{\prime}\right)\right| & =\prod_{j=1}^{t}\left|\mathrm{GL}\left(m, s_{j}\right)\right|=\prod_{j=1}^{t} q^{e_{j} d_{j} m^{2}} \prod_{i=1}^{m}\left(1-q^{-i d_{j}}\right) \\
& =q^{s m^{2}} \prod_{j=1}^{t} \prod_{i=1}^{m}\left(1-q^{-i d_{j}}\right)
\end{aligned}
$$

CoRotiaAiry 4.4. If $R_{m}=\left\{\sum_{i=0}^{n} a_{i} x^{l^{i}}: a_{i} \in \mathrm{GF}\left(q^{m}\right)\right\}$, then the group of units of $R_{m}$ is isomorphie to the diteot produet of the set $\left\{\mathrm{GL}\left(m, S_{j}\right)\right.$ : $j=1,2, \ldots, t\}$ of Theorern 4.3, and the order of the group of units of $R_{m}$. is given by (4.4).

ConoLxARY 4.5. Under the hypothesis of Theorem 4.3 , if also $(s, q)=1$, then the group of units of $R_{m}$ is isomorphic to a direat product of general linear groups. 
Proof. If $(s, q)=1$, then each exponent $e_{k}$ appearing in the factorization (4.3) of $x^{s}-1$ is equal to one, and each $s_{j}$ is isomorphic to the field $\operatorname{GF}\left(q^{l_{j}}\right)$. Then $\mathrm{GL}\left(m, S_{j}\right)=\mathrm{GL}\left(m, q^{d_{j}}\right)$ is a general linear group.

It should be noted that (4.4) can be derived directly from the result of Farahat [4] which gives the order of any finite ring $S$ with 1 in terms of $[$ rad $S]$ and the structure of $S /$ rad $S$ as assured by the WeddorburnArtin Theorem. This involves however computing $\mid$ rad $s^{\prime} \mid$ and knowing exactly how $S /$ rads decomposes into a direct sum of matrix rings over finite fields.

As final items we consider several interesting combinatorial questions. Suppose we are given the polynomial

$$
f(x)=\sum_{i=1}^{m-1} b_{i} x^{a^{i}} ; \quad b_{i} \in \operatorname{GTF}\left(q^{m}\right)
$$

so that $f(x)$ acting on $\mathrm{GF}\left(q^{m}\right)$ as a vector space over $\mathrm{GF}(q)$ is a linear transformation $f$. The questions are (i) How many $\varphi(x) \epsilon R_{n}$ when acting on GF $\left(q^{m}\right)$ equal $f$ and (ii) How many of these $p(x)$ are in the group of units of $R_{n}$; i.e., are permutations of $\mathrm{GF}\left(q^{n}\right)$. The answers to these questions are the content of our last theorem.

THEonem 4.6. The number of polynomials

$$
\varphi(x)=\sum_{i=1}^{n-\lambda} a_{i} x^{\alpha^{i}} ; \quad a_{i} \in \operatorname{GT}\left(q^{n}\right)
$$

whose restriction to $\mathrm{GF}\left(q^{m}\right)$ define the same functions as $(4.5)$ is $q^{n(n-m)}$. Of these, the number which are in the group of units of $R_{n}$ is zero if $f(x)$ is not one-one on $\mathrm{GF}\left(q^{m}\right)$ and is $q^{m(n-m)}|\mathrm{GL}(n-m, q)|$ if $f(x)$ is one-one on $\mathrm{GF}\left(q^{n}\right)$, where $|\mathrm{GL}(t, q)|$ is the well-known number $\prod_{i=0}^{t-1}\left(q^{l}-q^{i}\right)$. Thus, in particular, the number of such extensions of $f(x)$ is independent of the function $f(x)$.

Proof. Any $p(x)$ of the form (4.6) may be rewritten as

$$
\varphi(x)=\sum_{i=\infty}^{m-1} \sum_{j=1}^{8-1} a_{i+-m j} x^{i^{i+m j}} .
$$

\section{If $\xi \in \mathrm{GF}\left(q^{m}\right)$ then}

$$
\xi^{q^{i+1+m j}}=\xi^{q^{i}} \xi^{\alpha^{m j}}=\xi^{q^{i}}
$$

so that

$$
\varphi(\xi)=\sum_{i=0}^{m-1}\left(\sum_{j=0}^{s-1} a_{i+m j}\right) \xi^{q^{i}} .
$$

Hence

$$
\varphi(\xi)=f(\xi) \quad \text { for all } \xi \epsilon \mathrm{GF}\left(q^{m}\right)
$$

if and only if

$$
\sum_{j=0}^{s-1} a_{i \vdash-m j}=b_{i} \quad(i=0,1, \ldots, m-1) .
$$

The number of solutions $\left(a_{0}, a_{1}, \ldots, a_{n-1}\right)$ to this system of linear equations is independent of the particular $b_{i}$ 's and is $q^{n(s-1) m}=q^{n(n-n)}$ which completes the first part of the theorem.

As for the second part, clearly if $f(x)$ is not one-one on $\operatorname{GF}\left(q^{n}\right)$ none of the $p(x)$ maps on. $R_{n}$ whose restriction to $\mathrm{GF}\left(q^{m}\right)$ equals $f(x)$ can be one-one; thus, assume $f(x)$ is one-one on GF $\left(q^{m}\right)$. Any linear map is completely determined by itis action on a basis. Thus if $f(x)$ is given linear and one-one on $\mathrm{GE}\left(q^{m}\right)$, then the number of ways to extend $f(x)$ to a one-one linear map on $G \mathrm{~F}\left(q^{n}\right)$ is precisely the number of distinct ordered linearly independent sequences of $n-m$ elements of $\mathrm{GF}\left(q^{n}\right)$ which are bases for complementary subspaces of GF $\left(q^{m}\right)$. By a standard argument, this number is given by

$$
\begin{aligned}
\left(q^{n}-q^{m}\right)\left(q^{n}-q^{m+1}\right) & \cdots\left(q^{n}-q^{n-1}\right) \\
& =q^{n}\left(q^{n-m}-1\right) q^{m}\left(q^{n-m}-q\right) \ldots q^{m}\left(q^{n-m}-q^{n-m-1}\right) \\
& =q^{m(n-m)} \prod_{j=0}^{n-m-1}\left(q^{n-m}-q^{j}\right)=q^{m(n-m)}|\operatorname{GL}(n-m, q)| .
\end{aligned}
$$

This completes the proof.

\section{References}

[1] X. Carlitz, A note on the Betti-Mathieu group, Portugal. Math. 22 (1963), pp. 121-125.

[2] - and D. Frayos, Permutations with ooefficients in a subfield, Acta Arith. 21 (1972), pp, 31-35.

[3] L. Dickson, Linear Groups with an Fixposition of the Galois Field Theory, New Yorke 1958.

[4] H, K. Farahat, the multiplioative groups of a ring, Math. Zeitsohr. 87 (1965), pp. $378-384$.

[5] B. R. M. Donald, Involutary matrices over finite local rings, Canadian J, Math. 24 (1972), pp. $369-378$.

[6] 0. Oro, On a special lecss of polynomials, Amor. Mrath. Soc. Trazid. 35 (1933), I'p. $559-584$.

[7] - Oontributions is the the ry of finite Jelds, Amer. Math. Soc. Trans. 36 (1934), pp. 243-274. 